\title{
Enhancing Gamma Oscillations Restores Primary Motor Cortex Plasticity in Parkinson's Disease
}

\author{
${ }^{(1)}$ Andrea Guerra, ${ }^{1}{ }^{\circledR}$ Francesco Asci, ${ }^{2}$ Valentina D’Onofrio, ${ }^{2}$ Valerio Sveva, ${ }^{2}$ Matteo Bologna, ${ }^{1,2}$ \\ Giovanni Fabbrini, ${ }^{1,2}{ }^{\circledR}$ Alfredo Berardelli, ${ }^{1,2}$ and ${ }^{\circledR}$ Antonio Suppa ${ }^{1,2}$ \\ ${ }^{1}$ IRCCS Neuromed, Pozzilli (IS) 86077, Italy, and ${ }^{2}$ Department of Human Neurosciences, Sapienza University of Rome, Rome 00185, Italy
}

In humans, $\gamma$ oscillations in cortical motor areas reflect asynchronous synaptic activity and contribute to plasticity processes. In Parkinson's disease (PD), $\gamma$ oscillatory activity in the basal ganglia-thalamo-cortical network is altered and the LTP-like plasticity elicited by intermittent theta burst stimulation (iTBS) is reduced in the primary motor cortex (M1). In this study, we tested whether transcranial alternating current stimulation (tACS) delivered at $\gamma$ frequency promotes iTBS-induced LTPlike plasticity in M1 in PD patients. Sixteen patients (OFF condition) and 16 healthy subjects (HSs) underwent iTBS during $\gamma$-tACS (iTBS- $\gamma$ tACS) and during sham-tACS (iTBS-sham tACS) in two sessions. Motor-evoked potentials (MEPs) evoked by single-pulse transcranial magnetic stimulation and short-interval intracortical inhibition (SICI) were recorded before and after the costimulation. A subgroup of patients also underwent iTBS during $\beta$ tACS. iTBS-sham tACS facilitated single-pulse MEPs in HSs, but not in patients. iTBS- $\gamma$ tACS induced a larger MEP facilitation than iTBS-sham tACS in both groups, with similar values in patients and HSs. In patients, SICI improved after iTBS- $\gamma$ tACS. The effect produced by iTBS- $\gamma$ tACS on single-pulse MEPs correlated with disease duration, while changes in SICI correlated with Unified Parkinson's Disease Rating Scale Part III scores. The effect of iTBS- $\beta$ tACS on both single-pulse MEPs and SICI was similar to that obtained in the iTBS-sham tACS session. Our data suggest that $\gamma$ oscillations have a role in the pathophysiology of the abnormal LTP-like plasticity in PD. Entraining M1 neurons at the $\gamma$ rhythm through tACS may be an effective method to restore impaired plasticity.

Key words: gamma; motor cortex; Parkinson's disease; plasticity; tACS; TMS

Significance Statement

In Parkinson's disease, the LTP-like plasticity of the primary motor cortex is impaired, and $\gamma$ oscillations are altered in the basal ganglia-thalamo-cortical network. Using a combined transcranial magnetic stimulation-transcranial alternating current stimulation approach (iTBS- $\gamma$ tACS costimulation), we demonstrate that driving $\gamma$ oscillations restores the LTP-like plasticity in patients with Parkinson's disease. The effects correlate with clinical characteristics of patients, being more evident in less affected patients and weaker in patients with longer disease duration. These findings suggest that cortical $\gamma$ oscillations play a beneficial role in modulating the LTP-like plasticity of M1 in Parkinson's disease. The iTBS- $\gamma$ tACS approach may be potentially useful in rehabilitative settings in patients.

\section{Introduction}

In physiological conditions, neurophysiological recordings performed at the cortical level have demonstrated neuronal oscillations at the $\gamma$ frequency band, which are generated by the interaction between inhibitory interneurons and pyramidal neurons, reflecting intracortical synchronization processes (Traub et

\footnotetext{
Received Feb. 14, 2020; revised Mar. 26, 2020; accepted Apr. 26, 2020.

Author contributions: A.G., A.B., and A.S. designed research; A.G., F.A., V.D., and V.S. performed research; A.G. analyzed data; A.G. wrote the first draft of the paper; A.G. and A.S. wrote the paper; M.B. and G.F. contributed unpublished reagents/analytic tools; M.B., G.F., A.B., and A.S. edited the paper.

This work did not receive any specific grant from funding agencies in the public, commercial, or not-forprofit sectors. We thank all patients and healthy volunteers for their participation in this study.

The authors declare no competing financial interests.

Correspondence should be addressed to Alfredo Berardelli at alfredo.berardelli@uniroma1.it.

https://doi.org/10.1523/JNEUROSCI.0357-20.2020

Copyright $\odot 2020$ the authors
}

al., 2003; Bartos et al., 2007; Cardin et al., 2009; Otte et al., 2010; Giovanni et al., 2017). $\gamma$ oscillatory activity at $60-80 \mathrm{~Hz}$ is particularly relevant within the motor network, and its changes correlate with movement execution (Cassidy et al., 2002; Ball et al., 2008; Cheyne et al., 2008; Litvak et al., 2012). Experimental studies in animals and humans have also suggested that $\gamma$ oscillations promote synaptic plasticity in cortical motor areas (Diba and Buzsáki, 2007; Izaki and Akema, 2008; Girardeau et al., 2009; Nowak et al., 2017, 2018). In line with these observations, we have recently demonstrated that, in healthy subjects, transcranial alternating current stimulation (tACS), delivered over the cortical motor areas at $70 \mathrm{~Hz}$ together with intermittent theta burst stimulation (iTBS), promotes LTP-like plasticity in the primary motor cortex (M1) (Guerra et al., 2018b).

In patients with Parkinson's disease (PD), recent neurophysiological studies using iTBS demonstrated an impaired LTP-like 
plasticity in M1 (Suppa et al., 2011, 2016, 2017a,b; Udupa and Chen, 2013; Bologna et al., 2016), and neurophysiological recordings from basal ganglia nuclei and cortical motor areas demonstrated reduced $\gamma$ oscillations in the basal ganglia-thalamocortical network (Anzak et al., 2012; Florin et al., 2013; Jenkinson et al., 2013; Oswal et al., 2013; Little and Brown, 2014; Giovanni et al., 2017; Lofredi et al., 2018). Whether in patients with PD there is a relationship between the abnormal LTP-like plasticity in M1 and reduced $\gamma$ oscillations is unknown. We here hypothesize that enhancing $\gamma$ oscillations by means of $70 \mathrm{~Hz}$ tACS would promote processes of LTP-like plasticity in M1 in PD patients.

To explore this issue, we designed a single-blind, crossover, placebo-controlled study in which PD patients underwent iTBS during $\gamma$-tACS (iTBS- $\gamma$ tACS) and sham-tACS (iTBS-sham tACS) in two separate, randomized sessions. $\gamma$ tACS was delivered at $70 \mathrm{~Hz}$ since intracortical elements of M1 involved in motor control and plasticity processes have been shown to be particularly resonant at this specific high- $\gamma$ frequency (Nowak et al., 2017, 2018; Guerra et al., 2018b, 2019a; Bologna et al., 2019). We assessed the excitability of M1 before and after the combined stimulation by recording motor-evoked potentials (MEPs) evoked by single transcranial magnetic stimulation (TMS) pulses and short-interval intracortical inhibition (SICI), a paired-pulse TMS measure reflecting intracortical GABA-A-ergic inhibition (Kujirai et al., 1993; Di Lazzaro et al., 2007). SICI is known to be impaired in PD and is involved in brain plasticity mechanisms (Ridding et al., 1995; Berardelli et al., 2008; Teo et al., 2009; Di Lazzaro et al., 2018). Since our objective was to test the possible interaction between $\gamma$ oscillations, synaptic plasticity, and the disease per se, we assessed all patients OFF therapy. Also, to verify whether the effect of M1 oscillations on LTP-like plasticity was specific for the $\gamma$ frequency, a subgroup of patients underwent iTBS during tACS delivered at the $\beta$ frequency $(20 \mathrm{~Hz})$, a different cortical rhythm involved in motor control (Joundi et al., 2012; Oswal et al., 2013; Little and Brown, 2014; Guerra et al., 2016, 2018a; Giovanni et al., 2017; Bologna et al., 2019). Finally, in all participants, we verified the effects of $\gamma$-tACS (delivered alone) on M1 excitability by recording MEPs evoked by singlepulse TMS and SICI before, during and after tACS.

\section{Materials and Methods}

Participants

Sixteen PD patients (12 males; $67.8 \pm 9.9$ years; Table 1 ) and 16 healthy subjects (HSs; 9 males; $66.5 \pm 9.9$ years) participated. Patients were recruited from the Department of Human Neurosciences, Sapienza University (Rome). The diagnosis of PD was based on clinical criteria (Berardelli et al., 2013; Postuma et al., 2015). The clinical assessment included Hoehn \& Yahr Scale, the motor section of the Unified Parkinson's Disease Rating Scale Part III (UPDRS-III) (Goetz et al., 2008), Montreal Cognitive Assessment (MoCA) (Nasreddine et al., 2005), Frontal Assessment Battery (FAB) (Dubois et al., 2000), and Beck Depression Inventory Scale (BDI-II) (Beck et al., 1961). All participants were right-handed, as assessed by the Edinburgh Handedness Inventory (Oldfield, 1971). No subject had additional neuropsychiatric comorbidities or contraindications to the use of TMS or tACS (Rossi et al., 2009; Antal et al., 2017). No participant was taking drugs known to influence brain excitability or plasticity. The experimental procedures conformed to the Declaration of Helsinki, and all participants gave their informed consent to the study.

\section{TMS}

Single and paired-pulse TMS was performed using a MAGSTIM 200 connected to a figure-of-eight coil delivering monophasic pulses (Magstim). The optimal scalp position (hotspot) to elicit MEPs in the first dorsal interosseus muscle was assessed with the coil's handle
Table 1. Clinical-demographic characteristics of PD patientsa

\begin{tabular}{|c|c|c|c|c|c|c|c|c|c|c|}
\hline \multirow{2}{*}{$\begin{array}{l}\text { Subject } \\
\text { no. }\end{array}$} & \multirow{2}{*}{$\begin{array}{l}\text { Age } \\
(\mathrm{yr})\end{array}$} & \multirow[b]{2}{*}{ Gender } & \multirow{2}{*}{$\begin{array}{l}\text { Disease } \\
\text { duration (yr) }\end{array}$} & \multicolumn{2}{|c|}{ UPDRS-III } & \multirow[b]{2}{*}{ H\&Y } & \multirow[b]{2}{*}{$\mathrm{MoCA}$} & \multirow[b]{2}{*}{ FAB } & \multirow[b]{2}{*}{ BDI-II } & \multirow[b]{2}{*}{ LEDDs } \\
\hline & & & & Sham & $\gamma$ & & & & & \\
\hline 1 & 64 & M & 6 & 24 & 23 & 1 & 24 & 13 & 11 & 460 \\
\hline 2 & 61 & M & 3 & 23 & 26 & 2 & 26 & 18 & 13 & 860 \\
\hline 3 & 53 & M & 7 & 27 & 28 & 2 & 27 & 16 & 10 & 1000 \\
\hline 4 & 53 & M & 3 & 12 & 12 & 1 & 28 & 18 & 1 & 200 \\
\hline 5 & 75 & M & 5 & 45 & 44 & 2 & 26 & 15 & 17 & 700 \\
\hline 6 & 79 & M & 10 & 38 & 36 & 2 & 24 & 16 & 9 & 400 \\
\hline 7 & 76 & $\mathrm{~F}$ & 4 & 21 & 21 & 2 & 27 & 15 & 6 & 800 \\
\hline 8 & 64 & $\mathrm{~F}$ & 9 & 44 & 49 & 2 & 24 & 18 & 9 & 500 \\
\hline 9 & 69 & M & 1 & 11 & 10 & 1 & 29 & 17 & 5 & 200 \\
\hline 10 & 81 & M & 4 & 55 & 52 & 2 & 28 & 13 & 4 & 550 \\
\hline 11 & 65 & M & 6 & 28 & 29 & 2 & 30 & 16 & 3 & 605 \\
\hline 12 & 52 & M & 2 & 17 & 13 & 1 & 30 & 17 & 1 & 420 \\
\hline 13 & 67 & M & 10 & 40 & 42 & 2 & 29 & 16 & 7 & 700 \\
\hline 14 & 76 & M & 4 & 13 & 14 & 1 & 30 & 14 & 2 & 500 \\
\hline 15 & 68 & $\mathrm{~F}$ & 4 & 25 & 26 & 2 & 24 & 12 & 5 & 450 \\
\hline 16 & 82 & $\mathrm{~F}$ & 12 & 36 & 47 & 2 & 24 & 12 & 5 & 300 \\
\hline Mean & 67.8 & - & 5.6 & 28.7 & 29.5 & 1.7 & 26.9 & 15.4 & 6.7 & 540.3 \\
\hline SD & 9.9 & - & 3.2 & 13.1 & 14.0 & 0.5 & 2.4 & 2.1 & 4.5 & 227.7 \\
\hline
\end{tabular}

H\&Y, Hoehn \& Yahr Scale; LEDDs, L-Dopa equivalent daily doses.

pointing backward and laterally. The procedure was repeated twice: first, to center the tACS stimulating electrode over the hotspot; and second, after electrodes had been positioned over the scalp. Resting (rMTs) and active motor thresholds (AMTs) were then determined according to international guidelines (Rossini et al., 2015), along with the intensity that elicited MEPs of $\approx 1 \mathrm{mV}$ amplitude (MT1mV). MEPs were recorded using a pair of electrodes placed over the first dorsal interosseus (right side in HSs and most affected side in patients). EMG activity was amplified (Digitimer D360; Digitimer), digitized at $5 \mathrm{kHz}$ (CED 1401 interface; Cambridge Electronic Design), and stored on a computer for offline analyses (Signal software; Cambridge Electronic Design). A stereotaxic neuro-navigation system (SofTaxic Navigator System; EMS) was used to ensure accurate and reproducible positioning of the TMS coil over the identified hotspot.

SICI was tested according to standardized protocols (Kujirai et al., 1993; Berardelli et al., 2008). Paired pulses were delivered using an interstimulus interval of $2 \mathrm{~ms}$, with a conditioning stimulus intensity of $80 \%$ $\mathrm{AMT}$ and the test stimulus at MV1mV, so as to avoid possible contaminations by short-interval intracortical facilitation (Peurala et al., 2008). iTBS was delivered using a high-frequency biphasic magnetic stimulator (Magstim SuperRapid; Magstim). The stimulation protocol consisted of bursts of three pulses at $50 \mathrm{~Hz}$, repeated at $200 \mathrm{~ms}$ intervals $(5 \mathrm{~Hz})$. Bursts were delivered in short trains lasting $2 \mathrm{~s}$ with an $8 \mathrm{~s}$ pause between consecutive trains (20 trains, 600 total pulses). The stimulation intensity was set at 80\% AMT (Huang et al., 2005, 2017; Suppa et al., 2016). Since TBS elicits comparable MEP changes when delivered over the dominant and nondominant hemispheres in healthy subjects (Suppa et al., 2008), iTBS was always applied over the dominant hemisphere in the control group.

\section{tACS}

tACS was performed using conductive rubber electrodes $(5 \times 5 \mathrm{~cm})$ enclosed in sponges soaked with saline solution through a BrainSTIM (EMS). The stimulating electrode was centered over the first dorsal interosseus hotspot, while the reference electrode was placed over $\mathrm{Pz}$ (Guerra et al., 2016, 2018a,b, 2019a; Bologna et al., 2019), and secured in place using rubber strips around the head. Impedance was kept at $<10 \mathrm{k} \Omega$. tACS was delivered with no direct current off-set, a peak-to-peak amplitude of $1 \mathrm{~mA}$, and $3 \mathrm{~s}$ of ramping-up and ramping-down periods. Stimulation frequencies were set at $70 \mathrm{~Hz}(\gamma$-tACS $)$ and $20 \mathrm{~Hz}$ ( $\beta$-tACS), as in previous research demonstrating significant neurophysiological and behavioral effects on M1 (Joundi et al., 2012; Guerra et al., 2016, 2018a; Moisa et al., 2016; Bologna et al., 2019). Sham-tACS consisted of a short-lasting stimulation $(7 \mathrm{~s})$ at $70 \mathrm{~Hz}$. Accordingly, it 
A

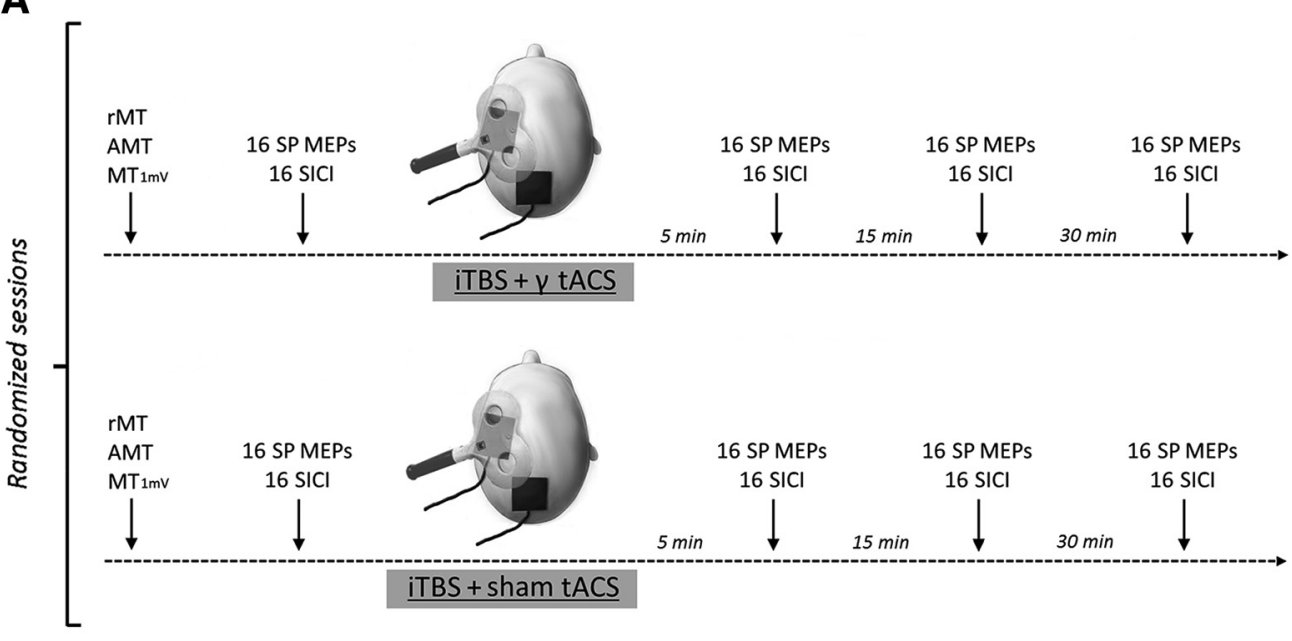

B

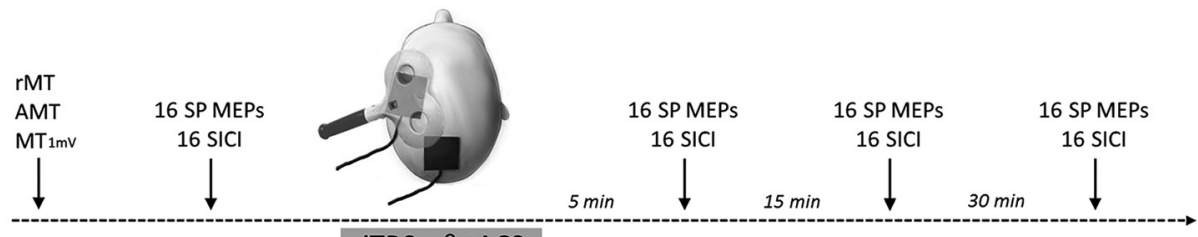

$\underline{\mathrm{TTBS}+\beta \mathrm{tACS}}$

C

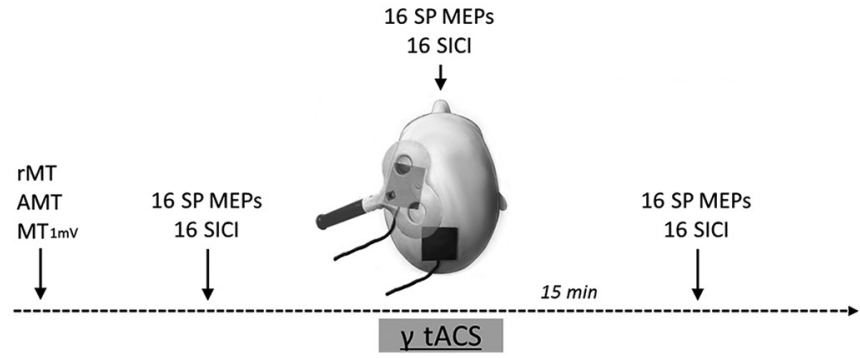

Figure 1. Experimental design. $\boldsymbol{A}$, Effects of iTBS- $\gamma$ tACS. Neurophysiological assessment started with the montage of tACS electrodes. Then, rMT, AMT, and the intensity able to induce an MT1mV were measured. In both iTBS- $\gamma$ tACS (top line) and iTBS-sham tACS (bottom line) sessions, 32 TMS stimuli (16 single pulses and 16 SICI) were randomly delivered before and 5, 15 , and $30 \mathrm{~min}$ after the costimulation. $\boldsymbol{B}$, Effects of iTBS- $\beta$ tACS. In a separate session involving a subgroup of patients, 16 single pulses and 16 SICI were randomly delivered before and 5 , 15 , and $30 \mathrm{~min}$ after iTBS- $\beta$ tACS. C, Effects of $\gamma$-tACS alone on M1 excitability. Sixteen single-pulse MEPs and 16 SICI were recorded before, during, and after $\gamma$-tACS.

terminated before the onset of iTBS in sham sessions. No participant reported visual or cutaneous sensations during tACS.

\section{Experimental design}

The experimental design (Fig. 1) consisted of three different sets of experiments performed on separate days.

Effects of iTBS- $\gamma$ tACS. All participants underwent two randomized sessions: (1) iTBS combined with $\gamma$-tACS (iTBS- $\gamma$ tACS); and (2) iTBS combined with sham-tACS (iTBS-sham tACS). Thirty-two TMS stimuli (16 single-pulse at MT1mV and 16 SICI) were randomly delivered before (T0), and 5 (T1), 15 (T2), and $30 \mathrm{~min}$ (T3) after iTBS. The interstimulus interval was $4.5-5.5 \mathrm{~s}$ to avoid habituation. tACS started $\approx 10 \mathrm{~s}$ before the application of the iTBS protocol; and in the iTBS- $\gamma$ tACS session, it was switched off immediately after the end of iTBS. Accordingly, iTBS- $\gamma$ tACS stimulation lasted $\approx 3$ min and $30 \mathrm{~s}$. iTBS-sham tACS and iTBS- $\gamma$ tACS sessions took place at comparable times of the day and were performed $\geq 7 \mathrm{~d}$ apart. All patients were studied in the OFF condition, at least $12 \mathrm{~h}$ after the intake of their usual dose of L-dopa.

Effects of iTBS- $\beta$ tACS. In a subgroup of 8 patients $(63.4 \pm$ 8.0 years), we tested the effects of iTBS combined with $\beta$-tACS (iTBS- $\beta$ tACS). In a separate session, 32 TMS stimuli (16 single-pulse at MT1mV and 16 SICI) were delivered at T0, T1, T2, and T3 after the costimulation. All patients were studied in the OFF condition.
Effects of $\gamma$-tACS alone on M1 excitability. In all participants, we tested the effect of $\gamma$-tACS alone (without iTBS) on corticospinal excitability: 16 single-pulse at MT1mV and 16 SICI were recorded before, during, and $15 \mathrm{~min}$ after tACS.

\section{Statistical analyses}

Peak-to-peak MEP amplitude was measured and averaged for each condition and time point. SICI was expressed as the ratio between the amplitude of MEPs evoked by paired-pulse TMS and the amplitude of single-pulse MEPs.

Differences in age, MoCA, BDI-II, and FAB between patients and HSs were evaluated using the Mann-Whitney $U$ test. Wilcoxon test was used to analyze differences in UPDRS-III scores in patients. Differences in gender distribution were evaluated using Fisher's exact test.

A repeated-measures ANOVA with group (levels: PD, HSs) and session (iTBS-sham tACS, iTBS- $\gamma$ tACS) as factors was used to compare AMT and rMT, as well as single-pulse MEPs and SICI at T0. To compare the effect of iTBS- $\gamma$ tACS in patients and HSs, we normalized singlepulse MEPs and SICI obtained after iTBS to pre-iTBS (T0) values. Two repeated-measures ANOVAs with group, session, and time point (levels: T1, T2, T3) as factors were then applied. To compare the effect of iTBS$\beta$ tACS with that induced by iTBS-sham tACS and iTBS- $\gamma$ tACS, we first verified possible differences in AMT, rMT, single-pulse MEPs, and 
SICI at T0 by using repeated-measures ANOVAs with session (levels: iTBS-sham tACS, iTBS- $\gamma$ tACS, iTBS- $\beta$ tACS) as factor. Then, we performed separate repeated-measures ANOVAs with session and time point as factors on single-pulse MEPs and SICI. Finally, to evaluate the effects of $\gamma$-tACS (alone) on single-pulse MEPs and SICI, we performed two repeated-measures ANOVAs with group and time point (levels: before, during, after tACS) as factors.

Greenhouse-Geisser corrections were applied when a violation of sphericity in Mauchly's tests was detected. Post hoc comparisons were performed using Bonferroni-corrected $t$ tests. The level of significance was set at $p<0.05$.

Pearson's correlation test was used to assess possible correlations in neurophysiological parameters. Spearman's rank-correlation test was applied to evaluate possible relationships between the effects of iTBS- $\gamma$ tACS and clinical-demographic characteristics of patients. For these purposes, we measured the overall extent of iTBS-tACS-induced changes on single-pulse MEPs and SICI (average of T1-T3). Then, synthetic measures that quantify the amount of modulation induced by iTBS- $\gamma$ tACS were created (i.e., ratio single-pulse iTBS- $\gamma$ tACS/sham tACS, ratio SICI iTBS- $\gamma$ tACS/sham tACS). Values are presented as mean \pm SD. Statistical analyses were performed using Statistica (StatSoft).

\section{Results}

No significant differences in age $(p=0.66)$, gender $(p=0.23)$, MoCA $(p=0.1)$, BDI-II $(p=0.12)$, or FAB scores $(p=0.16)$ were detected between PD patients and HSs. UPDRS-III scores were comparable in the two experimental sessions $(p=0.79)$. No study participant reported any adverse effect after combined iTBStACS stimulation.

\section{Pre-iTBS measures}

AMT (group: $F_{(1,30)}=0.01, p=0.92$; session: $F_{(1,30)}=0.14, p=$ 0.71 ; group $\times$ session: $F_{(1,30)}=1.06, p=0.31$ ), rMT (group: $F_{(1,30)}=3.02, p=0.09$; session: $F_{(1,30)}=2.92, p=0.1$; group $\times$ session: $\left.F_{(1,30)}=0.01, p=0.9\right)$, and single-pulse MEP amplitude at T0 (group: $F_{(1,30)}=0.25, p=0.62$; session: $F_{(1,30)}=0.24, p=0.63$; group $\times$ session: $\left.F_{(1,30)}=0.14, p=0.71\right)$ were similar in patients and HSs, in the two experimental sessions. Conversely, SICI at T0 differed between groups, being higher (weaker inhibition) in patients than HSs in both sessions (group: $F_{(1,30)}=4.62, p=0.04$, $\eta_{\mathrm{p}}{ }^{2}=0.13$; session: $F_{(1,30)}=2.89, p=0.1$; group $\times$ session: $F_{(1,30)}$ $=0.35, p=0.56)$.

\section{Effects of iTBS- $\gamma$ tACS}

When comparing the effects of iTBS- $\gamma$ tACS on single-pulse MEPs (Fig. 2, top), repeated-measures ANOVA disclosed a significant group $\times$ session interaction $\left(F_{(1,30)}=6.82, p=0.01, \eta_{\mathrm{p}}{ }^{2}\right.$ $=0.18)$. A significant effect of the factors session $\left(F_{(1,30)}=27.69\right.$, $\left.p<0.001, \eta_{\mathrm{p}}{ }^{2}=0.48\right)$ and time point $\left(F_{(2,60)}=3.42, p=0.04\right.$, $\left.\eta_{\mathrm{p}}{ }^{2}=0.10\right)$ also emerged, whereas the other factors or interactions were nonsignificant (group: $F_{(1,30)}=2.42, p=0.13$; group $\times$ time point: $F_{(2,60)}=0.37, p=0.69$; session $\times$ time point: $F_{(2,60)}=$ $0.19, p=0.83$; group $\times$ session $\times$ time point: $F_{(2,60)}=0.86, p=$ $0.43)$. Two separate between-group repeated-measures ANOVAs conducted for each session demonstrated that MEP facilitation after iTBS-sham tACS differed in PD patients and HSs, as shown by the significant factor group $\left(F_{(1,30)}=12.53, p<0.01, \eta_{\mathrm{p}}{ }^{2}=\right.$ $0.29)$ and the lack of group $\times$ time point interaction $\left(F_{(2,60)}=\right.$ $1.31, p=0.28)$. Post hoc analysis highlighted lower MEP facilitation in patients than HSs at T2 $(p=0.03)$ and T3 $(p=0.03)$. Conversely, the amount of facilitation after iTBS- $\gamma$ tACS was comparable between groups (group: $F_{(1,30)}=0.14, p=0.71$; group $\times$ time point: $\left.F_{(2,60)}=0.03, p=0.97\right)$. Notably, the extent of MEP facilitation after iTBS- $\gamma$ tACS was higher than that observed
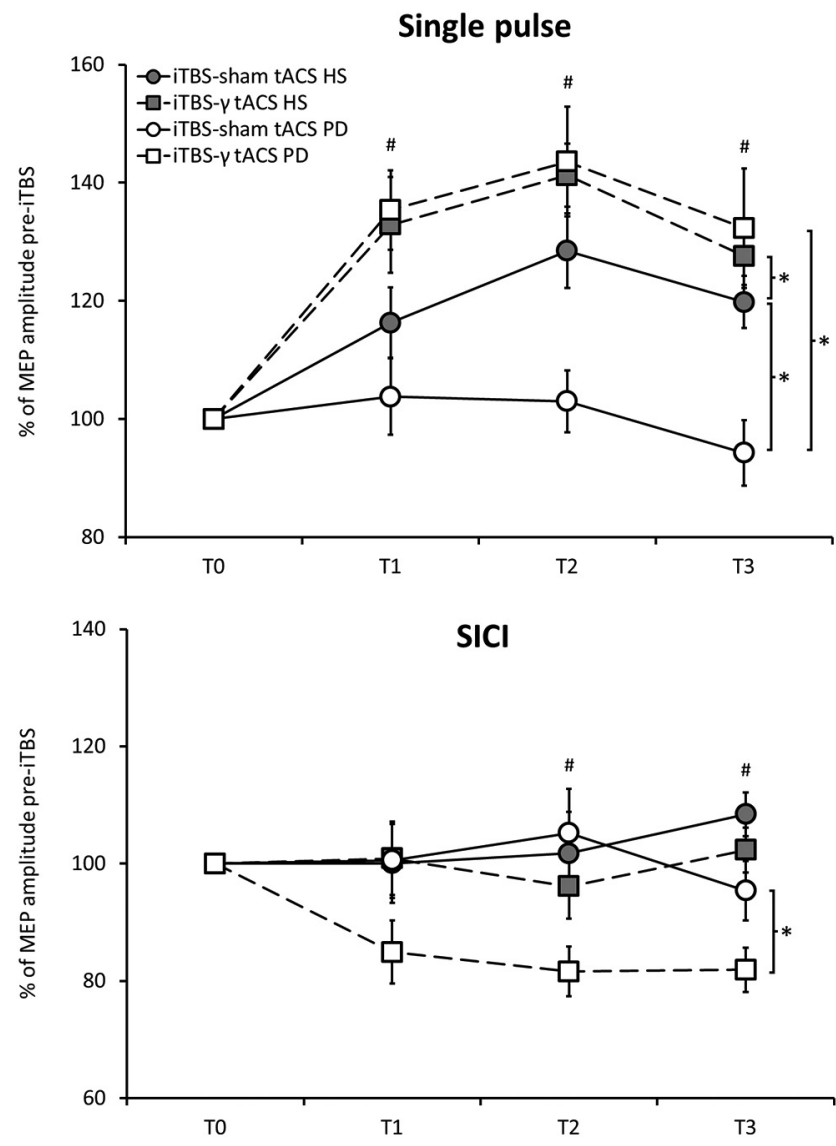

Figure 2. Effects of iTBS- $\gamma$ tACS costimulation. Top, Effect of iTBS- $\gamma$ tACS on MEPs evoked by single TMS pulses. A significantly greater facilitation of MEPs occurred after iTBS$\gamma$ tACS than after iTBS-sham tACS in both HSs and patients with PD. Of note, no MEP facilitation was present in the iTBS-sham tACS session in patients. Bottom, Effect of iTBS- $\gamma$ tACS on SICI. A significant reduction in SICI (greater inhibition) occurred after iTBS- $\gamma$ tACS than after iTBS-sham tACS in patients. MEP amplitudes (average \pm SEM) at 5 (T1), 15 (T2), and $30 \mathrm{~min}$ (T3) after iTBS are compared with pre-iTBS (T0) values (set as 100\%). *Significant differences between groups or sessions in the repeated-measures ANOVA. "Significant differences between iTBS- $\gamma$ tACS and iTBS-sham tACS at post hoc analyses of PD patients.

after iTBS-sham tACS in both groups, as confirmed by two repeated-measures ANOVAs conducted separately in HSs (session: $F_{(1,15)}=6.68, p=0.02, \eta_{\mathrm{p}}{ }^{2}=0.31$ ) and patients (session: $\left.F_{(1,15)}=21.03, p<0.001, \eta_{\mathrm{p}}{ }^{2}=0.58\right)$.

When comparing the effects of iTBS- $\gamma$ tACS on SICI (Fig. 2, bottom), the repeated-measures ANOVA revealed a significant group $\times$ session interaction $\left(F_{(1,30)}=5.56, p=0.02, \eta_{\mathrm{p}}{ }^{2}=0.16\right)$ and a significant factor session $\left(F_{(1,30)}=12.90, p<0.01, \eta_{\mathrm{p}}{ }^{2}=\right.$ $0.30)$. The effect of the factors group $\left(F_{(1,30)}=3.93, p=0.06\right)$ and time point $\left(F_{(2,60)}=0.03, p=0.97\right)$, as well as the group $\times$ time point $\left(F_{(2,60)}=1.59, p=0.21\right)$, session $\times$ time point $\left(F_{(2,60)}=\right.$ $0.64, p=0.53)$, and group $\times$ session $\times$ time point interactions $\left(F_{(2,60)}=0.39, p=0.68\right)$ were nonsignificant. Separate betweengroup repeated-measures ANOVAs demonstrated comparable changes in SICI in patients and HSs after iTBS-sham tACS (group: $F_{(1,30)}=0.19, p=0.66$; group $\times$ time point: $F_{(2,60)}=1.75$, $p=0.18)$. Conversely, SICI differed in patients and HSs after iTBS- $\gamma$ tACS (group: $F_{(1,30)}=14.12, p<0.001, \eta_{\mathrm{p}}{ }^{2}=0.32$; group $\times$ time point: $\left.F_{(2,60)}=0.22, p=0.8\right)$. Two additional repeatedmeasures ANOVAs clarified that, in HSs, SICI did not change after costimulation in either of the experimental sessions (session: $F_{(1,15)}=0.70, p=0.41$; session $\times$ time point: $F_{(2,30)}=0.28, p=$ 0.76). Conversely, in PD, SICI was differently modulated by 

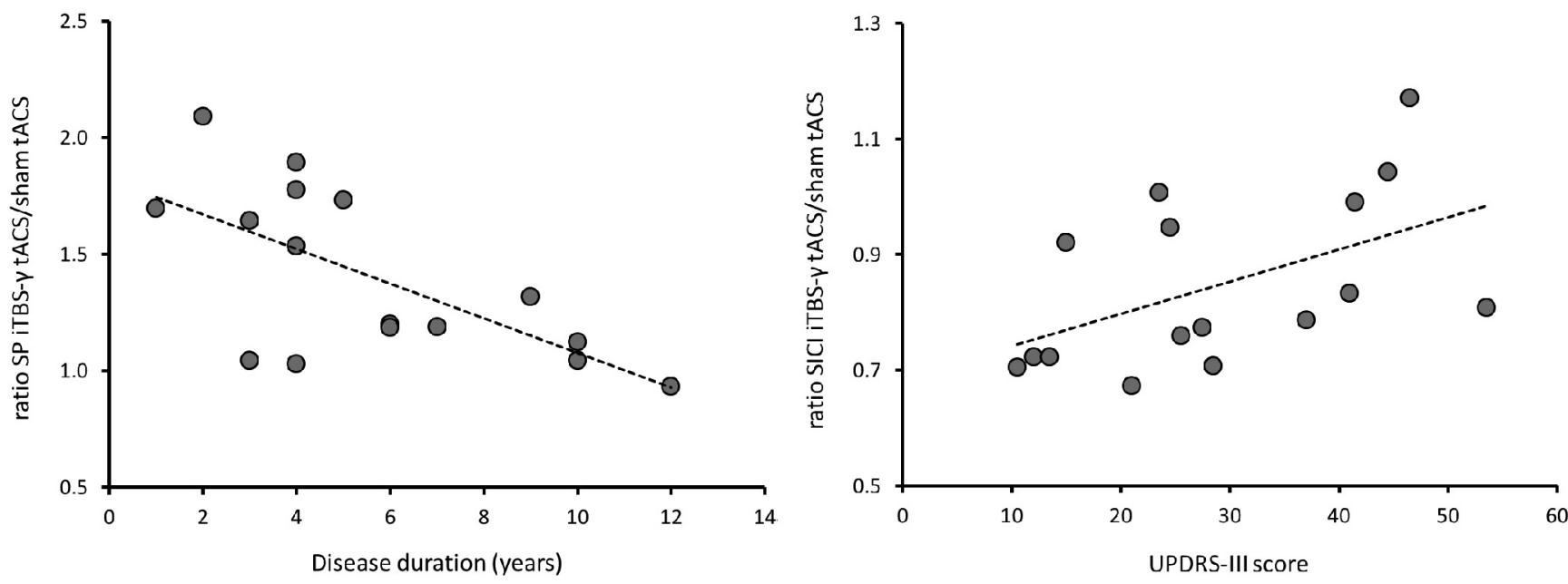

Figure 3. Clinical-neurophysiological correlations. Left, Negative correlation between the effect of iTBS- $\gamma$ tACS on single-pulse (SP) MEP amplitude (ratio SP iTBS- $\gamma$ tACS/sham tACS) and disease duration; that is, the greater the facilitation of MEPs after iTBS- $\gamma$ tACS, the shorter the disease duration. Right, Positive correlation between the effect of iTBS- $\gamma$ tACS on SICI (ratio SICI iTBS- $\gamma$ tACS/sham tACS) and the UPDRS-III score; that is, the greater the reduction (i.e., lower values) of SICI after iTBS- $\gamma$ tACS, the lower the UPDRS-III score.

iTBS-sham tACS and iTBS- $\gamma$ tACS (session: $F_{(1,15)}=19,22$, $p<0.001, \eta_{\mathrm{p}}{ }^{2}=0.56$; session $\times$ time point: $F_{(2,30)}=0.94, p=$ $0.40)$. In particular, SICI was not modified by iTBS-sham tACS (time point: $F_{(3,45)}=0.53, p=0.67$ ), whereas it decreased (greater inhibition) after iTBS- $\gamma$ tACS (time point: $F_{(3,45)}=5.41$, $p<0.01, \eta_{\mathrm{p}}{ }^{2}=0.26$ ), at T2 and T3 (T0 vs T1: $p=0.32$; T0 vs T2: $p<0.01$; T0 vs T3: $p<0.01)$.

\section{Clinical-neurophysiological correlations}

In patients, no correlation between the effects exerted by iTBS- $\gamma$ tACS on single-pulse MEPs and SICI was found ( $r=0.02, p=$ $0.9)$.

The effect produced by iTBS- $\gamma$ tACS on single-pulse MEPs negatively correlated with disease duration $(r=-0.55, p=0.02)$; that is, the longer the disease duration, the lower the MEP facilitation. Also, the effect exerted on SICI positively correlated with the UPDRS-III score (average of the two sessions; $r=0.57, p=$ $0.01)$; that is, the less severe the motor symptoms, the lower (greater inhibition) the SICI after iTBS- $\gamma$ tACS (Fig. 3).

\section{Effects of iTBS- $\beta$ tACS}

$\operatorname{AMT}\left(F_{(2,14)}=0.32, p=0.73\right), \operatorname{rMT}\left(F_{(2,14)}=0.03, p=0.89\right)$, single-pulse MEPs $\left(F_{(2,14)}=1.36, p=0.29\right)$, and SICI $\left(F_{(2,14)}=0.55\right.$, $p=0.59)$ at T0 were similar in iTBS-sham tACS, iTBS- $\gamma$ tACS, and iTBS- $\beta$ tACS sessions.

The effect of iTBS- $\beta$ tACS on both single-pulse MEPs and SICI was similar to that obtained in the iTBS-sham tACS session (singlepulse MEPs: frequency: $F_{(1,7)}=0.38, p=0.55$; frequency $\times$ time point: $F_{(2,14)}=2.49, p=0.12$; SICI: frequency: $F_{(1,7)}=0.77, p=0.41$; frequency $\times$ time point: $\left.F_{(2,14)}=0.99, p=0.39\right)$, and differed from that observed after iTBS- $\gamma$ tACS (single-pulse MEPs: frequency: $F_{(1,7)}=5.95, p=0.04$; frequency $\times$ time point: $F_{(2,14)}=0.31, p=$ 0.74 ; SICI: frequency: $F_{(1,7)}=5.44, p=0.05$; frequency $\times$ time point: $\left.F_{(2,14)}=1.09, p=0.36\right)$. These data overall suggest no aftereffects of iTBS- $\beta$ tACS on M1 excitability (Fig. 4).

\section{Effects of $\gamma$-tACS alone on M1 excitability}

In both PD patients and HSs, the amplitude of MEPs evoked by single-pulse TMS did not change during or after $\gamma$-tACS (time point: $F_{(2,60)}=2.33, p=0.11$; group: $F_{(1,30)}=0.54, p=0.47$; time point $\times$ group: $\left.F_{(2,60)}=0.1, p=0.9\right)$. Conversely, SICI was modulated by $\gamma$-tACS in both groups, as suggested by the significant

\section{Single pulse}
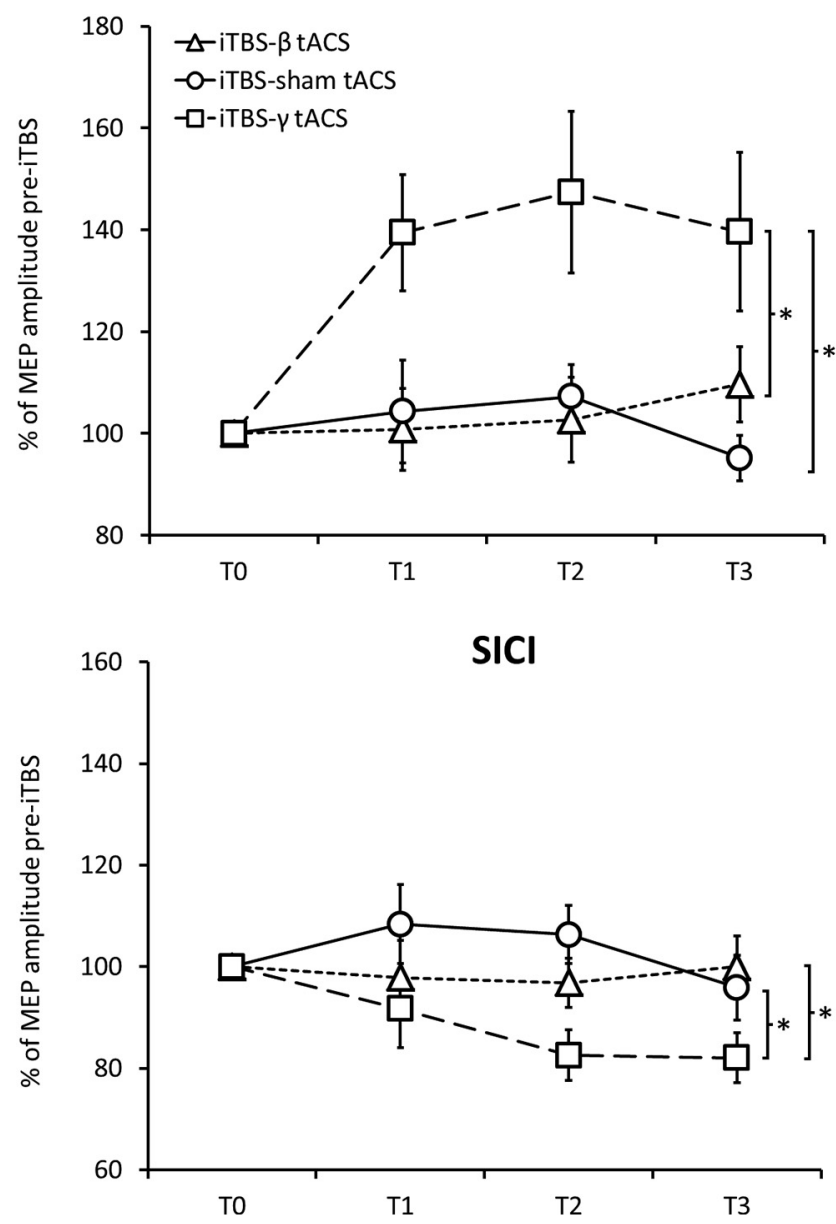

Figure 4. Effects of iTBS- $\beta$ tACS. iTBS- $\beta$ tACS did not change single-pulse MEP amplitude (top) or SICI (bottom). As a result, the effects of iTBS- $\beta$ tACS were comparable with those of iTBS-sham tACS and differed from those produced by iTBS- $\gamma$ tACS. * Significant differences between sessions.

factor time point $\left(F_{(2,60)}=6.74, p<0.01, \eta_{\mathrm{p}}{ }^{2}=0.18\right)$ and the lack of a time point $\times$ group interaction $\left(F_{(2,60)}=0.26, p=0.77\right)$. Post hoc analyses indicated higher SICI values during $\gamma$-tACS (during vs before tACS: $p=0.01$ ), an effect that disappeared 
when the stimulation ended (during vs after tACS: $p<0.01$; before vs after tACS: $p=0.99$ ).

\section{Discussion}

We have provided the first evidence suggesting that cortical $\gamma$ oscillations play a beneficial role in modulating the LTP-like plasticity of M1 in PD. Our data showed that iTBS-induced plasticity was impaired in the iTBS-sham tACS session in patients (no MEP facilitation after stimulation), whereas it returned to normal levels after iTBS- $\gamma$ tACS. Also, SICI, a neurophysiological measure altered in patients, became more effective (greater MEPs inhibition) after iTBS- $\gamma$ tACS. The effect of iTBS- $\gamma$ tACS on SICI was related to the severity of motor symptoms, whereas the improved iTBS-induced plasticity correlated with disease duration. iTBS- $\beta$ tACS did not modulate single-pulse MEP amplitude and SICI. Finally, $\gamma$-tACS delivered alone reduced SICI during stimulation but did not induce long-lasting changes in M1 excitability.

The main finding of our study is that impaired LTP-like plasticity of M1 in PD patients can be restored by using $\gamma$-tACS during the application of iTBS. Consistent with previous studies (Suppa et al., 2011, 2016, 2017a; Udupa and Chen, 2013; Bologna et al., 2016), in the iTBS-sham tACS session, we found a lack of LTP-like plasticity in M1 in patients. By comparing the effects of iTBS- $\gamma$ tACS on single-pulse MEPs with those produced by iTBS-sham tACS, we also confirmed that combined stimulation boosts the LTP-like plasticity of M1 in healthy subjects (Guerra et al., 2018b). More importantly, we showed that iTBS- $\gamma$ tACS improved LTP-like plasticity in PD patients, restoring it to normal levels. iTBS combined with $\beta$-tACS did not modulate M1 plasticity, thus suggesting that the effects produced by iTBStACS are frequency-specific. It is thought that tACS entrains the activity of "resonant" neuronal assemblies at the stimulating frequency, thus enhancing the power of the entrained cortical oscillations (Fröhlich and McCormick, 2010; Schutter and Hortensius, 2011; Reato et al., 2013). Accordingly, we propose that $\gamma$-tACS synchronized neuronal elements in M1 at the $\gamma$ frequency and boosted $\gamma$ oscillatory activity during the induction of plasticity through iTBS. Since we found that the application of $\gamma$-tACS alone did not increase corticospinal excitability, we believe that the improved plasticity resulted from a positive interaction between $\gamma$ oscillations, enhanced by tACS, and LTP-like plasticity mechanisms, activated by iTBS. In motor areas, $\gamma$ is considered a prokinetic rhythm. M1 neurons synchronize at the $\gamma$ frequency at $60-80 \mathrm{~Hz}$ during movement execution (Crone et al., 1998; Cheyne et al., 2008), and $\gamma$ tACS improves voluntary movement parameters in healthy subjects (Joundi et al., 2012; Moisa et al., 2016; Guerra et al., 2018a). Notably, animal (Diba and Buzsáki, 2007; Izaki and Akema, 2008; Girardeau et al., 2009) and human studies (Sederberg et al., 2003, 2007; Osipova et al., 2006; Nowak et al., 2017; Guerra et al., 2018b, 2019a) demonstrated that $\gamma$ activity is involved in brain plasticity processes. In $\mathrm{PD}$, cortical $\gamma$ oscillations are reduced in OFF (Bosboom et al., 2006; Stoffers et al., 2007) and increase in ON conditions (Williams et al., 2002; Lalo et al., 2008; Litvak et al., 2012). Accordingly, it has been suggested that $\gamma$ activity may be beneficial in PD by counteracting excessive antikinetic $\beta$ in the basal ganglia-thalamo-cortical network (Florin et al., 2013). Our findings indicate that the purported positive role played by $\gamma$ oscillations in PD also encompasses the facilitation of LTP-like plasticity processes. This effect may be mediated by $\gamma$-resonant neurons that are part of the motor network, such as GABA-Aergic fast-spiking parvalbumin-positive interneurons, which are known to contribute to the generation of $\gamma$ oscillations (Otte et al., 2010; Hu et al., 2014; Tremblay et al., 2016) and are also crucially involved in LTP-like plasticity (Benali et al., 2011; Suppa et al., 2016; Huang et al., 2017).

Another novel finding of our study concerns the effects produced by $\gamma$-tACS on SICI in patients. In keeping with previous studies (Berardelli et al., 2008; Bologna et al., 2018; Guerra et al., 2019b), we found that SICI, a measure reflecting GABA-A-ergic activity within M1 (Di Lazzaro et al., 2007), was less effective (reduced inhibition) in patients than controls. However, $\gamma$-tACS modulated SICI in both groups by decreasing inhibition during stimulation. This result agrees with studies on healthy subjects (Nowak et al., 2017; Guerra et al., 2018b, 2019a), but it is surprising to find it in PD since SICI was impaired at baseline. Accordingly, it may suggest that GABA-A-ergic M1 circuits resonant at $\gamma$ frequency are abnormal, though still functionally active in PD. Consistent with this hypothesis, we found that SICI became more effective in patients after iTBS- $\gamma$ tACS, suggesting enhanced GABA-A-ergic neurotransmission, in parallel with the improvement in LTP-like plasticity. This beneficial effect was present at 15 and $30 \mathrm{~min}$, but not at $5 \mathrm{~min}$ after stimulation. This may be due to the intrinsic variability of iTBS neurophysiological effects, which sometimes occur after several minutes and are generally more reliable $\approx 15 \mathrm{~min}$ after stimulation (Guerra et al., 2020a,b). Although the effect of iTBS- $\gamma$ tACS on SICI and that on single-pulse MEPs did not correlate, we hypothesize that the enhanced activity in $\gamma$-resonant GABA-A-ergic circuits plays a role in promoting LTPlike plasticity in PD. The opposite modulation of SICI found during $\gamma$-tACS (alone) and after iTBS- $\gamma$ tACS is not easy to explain but may reflect the involvement of different corticalsubcortical circuits. Indeed, tACS (alone) is thought to modulate neurons located in the superficial cortical layers, whereas the effect of tACS when combined with iTBS implies the activation of multiple circuits and a complex interaction between neurons located in different layers (Guerra et al., 2018b, 2019a). Therefore, the intracortical GABA-A-ergic activity may be differently modulated in the two conditions.

Importantly, the beneficial effects exerted by iTBS- $\gamma$ tACS correlated with some clinical characteristics of PD patients. The magnitude of the effect on SICI was positively related to the severity of motor symptoms (UPDRS-III scores); that is, the more severe the parkinsonian features, the less effective the SICI (i.e., higher values) after the costimulation. Also, the amount of improvement in the LTP-like plasticity of M1 was negatively associated with disease duration; that is, the longer the disease duration, the lower the enhancement in plasticity after the costimulation. The aforementioned correlations suggest that mechanisms underlying the beneficial effects of iTBS- $\gamma$ tACS are influenced by the severity of motor symptoms and deteriorate as the disease progresses. A possible hypothesis is that cortical neurons resonant at $\gamma$ rhythms, and, potentially responsible for improved LTP-like plasticity and GABA-A-ergic activity of M1, are sensitive to the neurodegeneration of the nigrostriatal pathway. Alternatively, these neurons may directly degenerate with disease progression. A further possibility is that $\gamma$ oscillations within the basal ganglia-thalamo-cortical network progressively decrease during the course of the disease. Accordingly, $\gamma$-tACS would be less effective in entraining $\gamma$ cortical activity in more advanced PD stages. 
When interpreting our results, it is important to note that the study design allowed us to exclude several confounding factors. MoCA, FAB, and BDI-II scores were similar in patients and HSs, allowing us to exclude that cognitive or depressive comorbidities could have biased our results. Baseline (pre-iTBS) levels of M1 excitability were comparable between patients and HSs as well as between iTBS-sham tACS and iTBS- $\gamma$ tACS sessions. ShamtACS was used as a placebo condition, and $\gamma$-tACS did not induce skin or visual sensations in any participant, thus ensuring that subjects were blinded to the stimulation's paradigm. A neuro-navigation system was used in all experiments to ensure a precise stimulation of the target throughout the assessments. The sessions were conducted at least 1 week apart, thus avoiding carryover effects. The UPDRS-III score was comparable between sessions, thus excluding any possible influence of motor symptoms on our results.

This study, however, has some limitations. First, we could not directly assess the effect of tACS on M1 $\gamma$ oscillations due to the presence of tACS artifact, which is several orders of magnitude greater than cortical activity. Also, our patients were stable responders to L-dopa, in the early-to-intermediate stage of the disease. Since previous evidence suggests that clinical characteristics of patients influence TBS-induced plasticity (Huang et al., 2011; Kishore et al., 2012), our results cannot be generalized to more advanced stages of PD. Furthermore, it is important to consider that the effect of $\gamma$-tACS (delivered alone or in combination with TBS) may be different if L-dopa-induced dyskinesia occurs, a clinical condition associated with specific intracortical abnormalities (Guerra et al., 2019b) and increased $\gamma$ oscillations in M1 (Dupre et al., 2016; Swann et al., 2016). Finally, the exact duration of iTBS- $\gamma$ tACS effects is unknown since no time point was assessed beyond $30 \mathrm{~min}$ after stimulation.

In conclusion, in this study, we provided the first evidence that boosting $\gamma$ oscillations through tACS during iTBS restores the LTP-like plasticity of M1 while simultaneously improving the impaired GABA-A-ergic neurotransmission in PD. These neurophysiological effects correlate with disease severity being more evident in less affected patients, and deteriorate as the disease progresses. The iTBS- $\gamma$ tACS costimulation may be potentially useful in rehabilitative settings to verify whether restoring brain plasticity enhances clinical motor outcomes in PD patients.

\section{References}

Antal A, Alekseichuk I, Bikson M, Brockmöller J, Brunoni AR, Chen R, Cohen LG, Dowthwaite G, Ellrich J, Flöel A, Fregni F, George MS, Hamilton R, Haueisen J, Herrmann CS, Hummel FC, Lefaucheur JP, Liebetanz D, Loo CK, McCaig CD, et al. (2017) Low intensity transcranial electric stimulation: safety, ethical, legal regulatory and application guidelines. Clin Neurophysiol 128:1774-1809.

Anzak A, Tan H, Pogosyan A, Foltynie T, Limousin P, Zrinzo L, Hariz M, Ashkan K, Bogdanovic M, Green AL, Aziz T, Brown P (2012) Subthalamic nucleus activity optimizes maximal effort motor responses in Parkinson's disease. Brain 135:2766-2778.

Ball T, Demandt E, Mutschler I, Neitzel E, Mehring C, Vogt K, Aertsen A, Schulze-Bonhage A (2008) Movement related activity in the high gamma range of the human EEG. Neuroimage 41:302-310.

Bartos M, Vida I, Jonas P (2007) Synaptic mechanisms of synchronized gamma oscillations in inhibitory interneuron networks. Nat Rev Neurosci 8:45-56.

Beck AT, Ward CH, Mendelson M, Mock J, Erbaugh J (1961) An inventory for measuring depression. Arch Gen Psychiatry 4:561-571.

Benali A, Trippe J, Weiler E, Mix A, Petrasch-Parwez E, Girzalsky W, Eysel UT, Erdmann R, Funke K (2011) Theta-burst transcranial magnetic stimulation alters cortical inhibition. J Neurosci 31:1193-1203.
Berardelli A, Wenning GK, Antonini A, Berg D, Bloem BR, Bonifati V, Brooks D, Burn DJ, Colosimo C, Fanciulli A, Ferreira J, Gasser T, Grandas F, Kanovsky P, Kostic V, Kulisevsky J, Oertel W, Poewe W, Reese JP, Relja M, et al. (2013) EFNS/MDS-ES recommendations for the diagnosis of Parkinson's disease. Eur J Neurol 20:16-34.

Berardelli A, Abbruzzese G, Chen R, Orth M, Ridding MC, Stinear C, Suppa A, Trompetto C, Thompson PD (2008) Consensus paper on short-interval intracortical inhibition and other transcranial magnetic stimulation intracortical paradigms in movement disorders. Brain Stimul 1:183-191.

Bologna M, Guerra A, Paparella G, Colella D, Borrelli A, Suppa A, Di Lazzaro V, Brown P, Berardelli A (2019) Transcranial alternating current stimulation has frequency-dependent effects on motor learning in healthy humans. Neuroscience 411:130-139.

Bologna M, Guerra A, Paparella G, Giordo L, Alunni Fegatelli D, Vestri AR, Rothwell JC, Berardelli A (2018) Neurophysiological correlates of bradykinesia in Parkinson's disease. Brain 141:2432-2444.

Bologna M, Suppa A, Conte A, Latorre A, Rothwell JC, Berardelli A (2016) Are studies of motor cortex plasticity relevant in human patients with Parkinson's disease? Clin Neurophysiol 127:50-59.

Bosboom JL, Stoffers D, Stam CJ, van Dijk BW, Verbunt J, Berendse HW, Wolters EC (2006) Resting state oscillatory brain dynamics in Parkinson's disease: an MEG study. Clin Neurophysiol 117:2521-2531.

Cardin JA, Carlén M, Meletis K, Knoblich U, Zhang F, Deisseroth K, Tsai LH, Moore CI (2009) Driving fast-spiking cells induces gamma rhythm and controls sensory responses. Nature 459:663-667.

Cassidy M, Mazzone P, Oliviero A, Insola A, Tonali P, Di Lazzaro V, Brown $P$ (2002) Movement-related changes in synchronization in the human basal ganglia. Brain 125:1235-1246.

Cheyne D, Bells S, Ferrari P, Gaetz W, Bostan AC (2008) Self-paced movements induce high-frequency gamma oscillations in primary motor cortex. Neuroimage 42:332-342.

Crone NE, Miglioretti DL, Gordon B, Lesser RP (1998) Functional mapping of human sensorimotor cortex with electrocorticographic spectral analysis: II. Event-related synchronization in the gamma band. Brain 121:2301-2315.

Di Lazzaro V, Pilato F, Dileone M, Profice P, Ranieri F, Ricci V, Bria P, Tonali PA, Ziemann U (2007) Segregating two inhibitory circuits in human motor cortex at the level of GABAA receptor subtypes: a TMS study. Clin Neurophysiol 118:2207-2214.

Di Lazzaro V, Rothwell J, Capogna M (2018) Noninvasive stimulation of the human brain: activation of multiple cortical circuits. Neuroscientist 24:246-260.

Diba K, Buzsáki G (2007) Forward and reverse hippocampal place-cell sequences during ripples. Nat Neurosci 10:1241-1242.

Dubois B, Slachevsky A, Litvan I, Pillon B (2000) The FAB: a Frontal Assessment Battery at bedside. Neurology 55:1621-1626.

Dupre KB, Cruz AV, McCoy AJ, Delaville C, Gerber CM, Eyring KW, Walters JR (2016) Effects of L-dopa priming on cortical high beta and high gamma oscillatory activity in a rodent model of Parkinson's disease. Neurobiol Dis 86:1-15.

Florin E, Erasmi R, Reck C, Maarouf M, Schnitzler A, Fink GR, Timmermann L (2013) Does increased gamma activity in patients suffering from Parkinson's disease counteract the movement inhibiting beta activity? Neuroscience 237:42-50.

Fröhlich F, McCormick DA (2010) Endogenous electric fields may guide neocortical network activity. Neuron 67:129-143.

Giovanni A, Capone F, di Biase L, Ferreri F, Florio L, Guerra A, Marano M, Paolucci M, Ranieri F, Salomone G, Tombini M, Thut G, Di Lazzaro V (2017) Oscillatory activities in neurological disorders of elderly: biomarkers to target for neuromodulation. Front Aging Neurosci 9:189.

Girardeau G, Benchenane K, Wiener SI, Buzsáki G, Zugaro MB (2009) Selective suppression of hippocampal ripples impairs spatial memory. Nat Neurosci 12:1222-1223.

Goetz CG, Tilley BC, Shaftman SR, Stebbins GT, Fahn S, Martinez-Martin P, Poewe W, Sampaio C, Stern MB, Dodel R, Dubois B, Holloway R, Jankovic J, Kulisevsky J, Lang AE, Lees A, Leurgans S, LeWitt PA, Nyenhuis D, Olanow CW, et al. (2008) Movement Disorder Societysponsored revision of the Unified Parkinson's Disease Rating Scale (MDS-UPDRS): scale presentation and clinimetric testing results. Mov Disord 23:2129-2170.

Guerra A, Pogosyan A, Nowak M, Tan H, Ferreri F, Di Lazzaro V, Brown P (2016) Phase dependency of the human primary motor cortex and 
cholinergic inhibition cancelation during beta tACS. Cereb Cortex 26:3977-3990.

Guerra A, Bologna M, Paparella G, Suppa A, Colella D, Di Lazzaro V, Brown P, Berardelli A (2018a) Effects of transcranial alternating current stimulation on repetitive finger movements in healthy humans. Neural Plast 2018:4593095

Guerra A, Suppa A, Bologna M, D’Onofrio V, Bianchini E, Brown P, Di Lazzaro V, Berardelli A (2018b) Boosting the LTP-like plasticity effect of intermittent theta-burst stimulation using gamma transcranial alternating current stimulation. Brain Stimul 11:734-742.

Guerra A, Suppa A, Asci F, De Marco G, D’Onofrio V, Bologna M, Di Lazzaro V, Berardelli A (2019a) LTD-like plasticity of the human primary motor cortex can be reversed by $\gamma$-tACS. Brain Stimul 12:1490-1499.

Guerra A, Suppa A, D’Onofrio V, Di Stasio F, Asci F, Fabbrini G, Berardelli A (2019b) Abnormal cortical facilitation and L-dopa-induced dyskinesia in Parkinson's disease. Brain Stimul 12:1517-1525.

Guerra A, López-Alonso V, Cheeran B, Suppa A (2020a) Variability in noninvasive brain stimulation studies: reasons and results. Neurosci Lett 719:133330.

Guerra A, López-Alonso V, Cheeran B, Suppa A (2020b) Solutions for managing variability in non-invasive brain stimulation studies. Neurosci Lett 719:133332.

$\mathrm{Hu}$ H, Gan J, Jonas P (2014) Interneurons. Fast-spiking, parvalbumin ${ }^{+}$ GABAergic interneurons: from cellular design to microcircuit function. Science 345:1255263.

Huang YZ, Edwards MJ, Rounis E, Bhatia KP, Rothwell JC (2005) Theta burst stimulation of the human motor cortex. Neuron 45:201-206.

Huang YZ, Rothwell JC, Lu CS, Chuang WL, Chen RS (2011) Abnormal bidirectional plasticity-like effects in Parkinson's disease. Brain 134:23122320.

Huang YZ, Lu MK, Antal A, Classen J, Nitsche M, Ziemann U, Ridding M, Hamada M, Ugawa Y, Jaberzadeh S, Suppa A, Paulus W, Rothwell J (2017) Plasticity induced by non-invasive transcranial brain stimulation: a position paper. Clin Neurophysiol 128:2318-2329.

Izaki Y, Akema T (2008) Gamma-band power elevation of prefrontal local field potential after posterior dorsal hippocampus-prefrontal long-term potentiation induction in anesthetized rats. Exp Brain Res 184:249-253.

Jenkinson N, Kühn AA, Brown P (2013) $\gamma$ oscillations in the human basal ganglia. Exp Neurol 245:72-76.

Joundi RA, Jenkinson N, Brittain JS, Aziz TZ, Brown P (2012) Driving oscillatory activity in the human cortex enhances motor performance. Curr Biol 22:403-407.

Kishore A, Popa T, Velayudhan B, Joseph T, Balachandran A, Meunier S (2012) Acute dopamine boost has a negative effect on plasticity of the primary motor cortex in advanced Parkinson's disease. Brain 135:20742088.

Kujirai T, Caramia MD, Rothwell JC, Day BL, Thompson PD, Ferbert A, Wroe S, Asselman P, Marsden CD (1993) Corticocortical inhibition in human motor cortex. J Physiol 471:501-519.

Lalo E, Thobois S, Sharott A, Polo G, Mertens P, Pogosyan A, Brown P (2008) Patterns of bidirectional communication between cortex and basal ganglia during movement in patients with Parkinson disease. J Neurosci 28:3008-3016

Little S, Brown P (2014) The functional role of beta oscillations in Parkinson's disease. Parkinsonism Relat Disord 20[Suppl 1]:S44-S48.

Litvak V, Eusebio A, Jha A, Oostenveld R, Barnes G, Foltynie T, Limousin P, Zrinzo L, Hariz MI, Friston K, Brown P (2012) Movement-related changes in local and long-range synchronization in Parkinson's disease revealed by simultaneous magnetoencephalography and intracranial recordings. J Neurosci 32:10541-10553.

Lofredi R, Neumann WJ, Bock A, Horn A, Huebl J, Siegert S, Schneider GH, Krauss JK, Kühn AA (2018) Dopamine-dependent scaling of subthalamic gamma bursts with movement velocity in patients with Parkinson's disease. Elife 7:e31895.

Moisa M, Polania R, Grueschow M, Ruff CC (2016) Brain network mechanisms underlying motor enhancement by transcranial entrainment of gamma oscillations. J Neurosci 36:12053-12065.

Nasreddine ZS, Phillips NA, Bédirian V, Charbonneau S, Whitehead V, Collin I, Cummings JL, Chertkow H (2005) The Montreal Cognitive Assessment, MoCA: a brief screening tool for mild cognitive impairment. J Am Geriatr Soc 53:695-699.
Nowak M, Hinson E, van Ede F, Pogosyan A, Guerra A, Quinn A, Brown P, Stagg CJ (2017) Driving human motor cortical oscillations leads to behaviorally relevant changes in local GABAA inhibition: a tACS-TMS study. J Neurosci 37:4481-4492.

Nowak M, Zich C, Stagg CJ (2018) Motor cortical gamma oscillations: what have we learnt and where are we headed? Curr Behav Neurosci Rep 5:136-142.

Oldfield RC (1971) The assessment and analysis of handedness: the Edinburgh Inventory. Neuropsychologia 9:97-113.

Osipova D, Takashima A, Oostenveld R, Fernández G, Maris E, Jensen O (2006) Theta and gamma oscillations predict encoding and retrieval of declarative memory. J Neurosci 26:7523-7531.

Oswal A, Brown P, Litvak V (2013) Synchronized neural oscillations and the pathophysiology of Parkinson's disease. Curr Opin Neurol 26:662-670.

Otte S, Hasenstaub A, Callaway EM (2010) Cell type-specific control of neuronal responsiveness by gamma-band oscillatory inhibition. J Neurosci 30:2150-2159.

Peurala SH, Müller-Dahlhaus JF, Arai N, Ziemann U (2008) Interference of short-interval intracortical inhibition (SICI) and short-interval intracortical facilitation (SICF). Clin Neurophysiol 119:2291-2297.

Postuma RB, Berg D, Stern M, Poewe W, Olanow CW, Oertel W, Obeso J, Marek K, Litvan I, Lang AE, Halliday G, Goetz CG, Gasser T, Dubois B, Chan P, Bloem BR, Adler CH, Deuschl G (2015) MDS clinical diagnostic criteria for Parkinson's disease. Mov Disord 30:1591-1601

Reato D, Rahman A, Bikson M, Parra LC (2013) Effects of weak transcranial alternating current stimulation on brain activity: a review of known mechanisms from animal studies. Front Hum Neurosci 7:687.

Ridding MC, Inzelberg R, Rothwell JC (1995) Changes in excitability of motor cortical circuitry in patients with Parkinson's disease. Ann Neurol 37:181-188

Rossi S, Hallett M, Rossini PM, Pascual-Leone A, Safety of TMS Consensus Group (2009) Safety, ethical considerations, and application guidelines for the use of transcranial magnetic stimulation in clinical practice and research. Clin Neurophysiol 120:2008-2039.

Rossini PM, Burke D, Chen R, Cohen LG, Daskalakis Z, Di Iorio R, Di Lazzaro V, Ferreri F, Fitzgerald PB, George MS, Hallett M, Lefaucheur JP, Langguth B, Matsumoto H, Miniussi C, Nitsche MA, Pascual-Leone A, Paulus W, Rossi S, Rothwell JC, et al. (2015) Non-invasive electrical and magnetic stimulation of the brain, spinal cord, roots and peripheral nerves: basic principles and procedures for routine clinical and research application. An updated report from an I.F.C.N. Committee. Clin Neurophysiol 126:1071-1107.

Schutter D, Hortensius R (2011) Brain oscillations and frequency-dependent modulation of cortical excitability. Brain Stimul 4:97-103.

Sederberg PB, Kahana MJ, Howard MW, Donner EJ, Madsen JR (2003) Theta and gamma oscillations during encoding predict subsequent recall. J Neurosci 23:10809-10814.

Sederberg PB, Schulze-Bonhage A, Madsen JR, Bromfield EB, McCarthy DC, Brandt A, Tully MS, Kahana MJ (2007) Hippocampal and neocortical gamma oscillations predict memory formation in humans. Cereb Cortex 17:1190-1196.

Stoffers D, Bosboom JL, Deijen JB, Wolters EC, Berendse HW, Stam CJ (2007) Slowing of oscillatory brain activity is a stable characteristic of Parkinson's disease without dementia. Brain 130:1847-1860.

Suppa A, Ortu E, Zafar N, Deriu F, Paulus W, Berardelli A, Rothwell JC (2008) Theta burst stimulation induces after-effects on contralateral primary motor cortex excitability in humans. J Physiol 586:4489-4500.

Suppa A, Marsili L, Belvisi D, Conte A, Iezzi E, Modugno N, Fabbrini G, Berardelli A (2011) Lack of LTP-like plasticity in primary motor cortex in Parkinson's disease. Exp Neurol 227:296-301.

Suppa A, Huang YZ, Funke K, Ridding MC, Cheeran B, Di Lazzaro V, Ziemann U, Rothwell JC (2016) Ten years of theta burst stimulation in humans: established knowledge, unknowns and prospects. Brain Stimul 9:323-335.

Suppa A, Bologna M, Conte A, Berardelli A, Fabbrini G (2017a) The effect of L-dopa in Parkinson's disease as revealed by neurophysiological studies of motor and sensory functions. Expert Rev Neurother 17:181-192. 
Suppa A, Quartarone A, Siebner H, Chen R, Di Lazzaro V, Del Giudice P, Paulus W, Rothwell JC, Ziemann U, Classen J (2017b) The associative brain at work: evidence from paired associative stimulation studies in humans. Clin Neurophysiol 128:2140-2164.

Swann NC, de Hemptinne C, Miocinovic S, Qasim S, Wang SS, Ziman N, Ostrem JL, San Luciano M, Galifianakis NB, Starr PA (2016) Gamma oscillations in the hyperkinetic state detected with chronic human brain recordings in Parkinson's disease. J Neurosci 36:6445-6458.

Teo JT, Terranova C, Swayne O, Greenwood RJ, Rothwell JC (2009) Differing effects of intracortical circuits on plasticity. Exp Brain Res 193:555-563.
Traub RD, Pais I, Bibbig A, LeBeau FE, Buhl EH, Hormuzdi SG, Monyer H, Whittington MA (2003) Contrasting roles of axonal (pyramidal cell) and dendritic (interneuron) electrical coupling in the generation of neuronal network oscillations. Proc Natl Acad Sci USA 100:1370-1374.

Tremblay R, Lee S, Rudy B (2016) GABAergic interneurons in the neocortex: from cellular properties to circuits. Neuron 91:260-292.

Udupa K, Chen R (2013) Motor cortical plasticity in Parkinson's disease. Front Neurol 4:128.

Williams D, Tijssen M, Van Bruggen G, Bosch A, Insola A, Di Lazzaro V, Mazzone P, Oliviero A, Quartarone A, Speelman H, Brown P (2002) Dopamine-dependent changes in the functional connectivity between basal ganglia and cerebral cortex in humans. Brain 125:1558-1569. 